\title{
Levamisole-induced and COVID-19-induced retiform purpura: two overlapping, emerging clinical syndromes
}

\author{
Catherine K. Keim ${ }^{1} \cdot$ Robert A. Schwartz ${ }^{2} \cdot$ Rajendra Kapila $^{3}$ \\ Received: 10 July 2021 / Accepted: 11 November 2021 / Published online: 22 November 2021 \\ (c) The Author(s), under exclusive licence to Springer-Verlag GmbH Germany, part of Springer Nature 2021
}

\begin{abstract}
Levamisole exposure in cocaine users is a well-recognized cause of retiform purpura, a distinctive net-like maculopapular patch. Prolonged exposure to levamisole can lead to a serious systemic syndrome known as levamisole-induced vasculitis, most commonly involving the kidneys and lungs. More recently, retiform purpura has been observed in patients with the novel coronavirus disease of 2019 (COVID-19). Due to their overlapping dermatologic and systemic manifestations, levamisoleinduced and COVID-19-induced retiform purpura may mimic one another in clinical presentation. The possibility that patients may present with one or both syndromes creates a diagnostic challenge. This review of levamisole-induced and COVID19-induced retiform purpura highlights their corresponding and distinctive features. Additionally, we propose a unique staging system for levamisole-induced retiform purpura that may be valid for future classification of COVID-19-induced retiform purpura.
\end{abstract}

Keywords Levamisole $\cdot$ Cocaine $\cdot$ Vasculitis $\cdot$ Retiform purpura $\cdot$ COVID-19 $\cdot$ Neutrophils $\cdot$ Drug abuse

\section{Introduction}

Over the last 10 years, retiform purpura has been extensively observed in patients exposed to levamisole-contaminated cocaine. While patients are often first evident with cutaneous manifestations, end-organ disease may occur as well. More recently, retiform purpura has been observed in patients presenting with the coronavirus disease of 2019 (COVID-19).

Levamisole is an immunomodulatory agent previously employed to treat autoimmune disorders and

Robert A. Schwartz

raschwartz@gmail.com

Catherine K. Keim

ckk36@njms.rutgers.edu

Rajendra Kapila

dsax.kap@gmail.com

1 Rutgers New Jersey Medical School, MSB H-576, 185 South Orange Ave, Newark, NJ 07103, USA

2 Dermatology and Pathology, Rutgers New Jersey Medical School, MSB H-576, 185 South Orange Ave, Newark, NJ 07103, USA

3 Infectious Diseases, Rutgers New Jersey Medical School, MSB H-576, 185 South Orange Ave, Newark, NJ 07103, USA malignancies. Its mechanism of action is thought to involve neutrophil stimulation and T-cell proliferation [1]. Due to adverse effects, most notably severe neutropenia, levamisole was banned from US markets in 1999. Since then, levamisole has gained recognition as an additive in cocaine. Aminorex, an active metabolite of levamisole, has a similar potency and mechanism of action to cocaine [2, 3]. Levamisole's current use in veterinary medicine makes it widely accessible. Its physical properties make it virtually indistinguishable from cocaine. Levamisole is therefore able to potentiate the effects of cocaine while reducing costs to producers. According to the DEA, in 2009, approximately $71 \%$ of cocaine in the US was contaminated with levamisole, and by October 2017, that number had increased to $87 \%[3,4]$.

\section{Literature review}

The adverse effects of levamisole-contaminated cocaine were first reported in a case series of unexplained agranulocytosis in cocaine users in 2009 [5]. In 2011, the first cases of levamisole-induced retiform purpura were described [6]. Patients presented with a net-like, or retiform, purpura progressing to necrosis after exposure to 
levamisole-contaminated cocaine. In subsequent cases, patients presented with multiorgan involvement including acute kidney injury, alveolar hemorrhage, and pneumonia [7]. Since then, levamisole-contaminated cocaine has become a well-described cause of a vasculitis characterized by retiform purpura with or without end-organ involvement.

COVID-19 is a predominantly respiratory syndrome caused by the novel severe acute respiratory syndrome coronavirus type-2 (SARS-CoV-2). It was first reported from Wuhan, China in December 2019. By March 2020, it had been declared a pandemic by the World Health Organization (WHO) [8]. Manifestations of COVID-19 range from asymptomatic carriers, to respiratory disease, to multiorgan dysfunction and failure [9]. As the disease has progressed, the cardiac, gastrointestinal, renal, and neurologic manifestations of COVID-19 have been described [9].

More recently, COVID-19 has been linked to a variety of dermatologic manifestations [10, 11]. Suchonwanit et al. [12] categorized cutaneous lesions in COVID-19 into two major mechanistic patterns: (1) clinical features similar to viral exanthems, or (2) lesions secondary to systemic consequences of COVID-19, especially vasculitis and thrombotic vasculopathy. Within the category of thrombotic vasculopathy, retiform purpura has been described [13].

The overlap between the cutaneous and systemic symptoms of levamisole-induced and COVID-19-induced retiform purpura creates the potential for these two syndromes to mimic one another in clinical presentation. As the coronavirus pandemic progresses, the number of cases continues to rise [14]. At the same time, the unique psychosocial stressors created by the pandemic have the potential to increase rates of substance abuse among current users and the general population [15]. Not only is substance abuse a risk factor for contracting COVID-19, but it is also associated with greater morbidity and mortality due to the virus than is seen in the general population [16]. The resulting possibility that patients may present with one or both of these syndromes creates a diagnostic challenge with clinical implications.

This review of levamisole-induced and COVID-19-induced retiform purpura highlights their corresponding and distinctive features. Additionally, we propose a unique staging system for levamisole-induced retiform purpura that may be valid for future classification of COVID-19-induced retiform purpura.

\section{Epidemiology and clinical presentation of levamisole-induced retiform purpura}

\section{Epidemiology}

Cutaneous symptoms occur in $0.5-3 \%$ of those exposed to levamisole-contaminated cocaine and typically appear $1-4$ days after use [17]. Lesions occur regardless of the route of cocaine administration and progress in a dose-dependent manner [17-19]. Incidence is higher in women and in individuals with HLA B27 genotype or preexisting rheumatic disease $[17,20]$. The average age of onset is $42 \cdot 7$ years [17].

\section{Clinical presentation}

The classic presentation is a net-like, violaceous patch (Fig. 1). Lesions are typically bilateral, symmetric, and painful. Upon continued exposure to levamisole, lesions may progress to ulcerating bullae, skin sloughing, and necrosis (Fig. 2). Skin necrosis may become so severe that underlying soft tissue and bone become exposed (Fig. 3) [17]. Though the pathognomonic site of involvement is the pinna of the ear, the most common sites of involvement in decreasing prevalence are the face and lower extremities, ears, upper extremities, and trunk [7]. Oropharyngeal mucosal patches may also occur [21].

Based on the severity of skin manifestations, we propose a unique staging system for levamisole-induced retiform purpura summarized in Table 1. Lesions in stage I do not

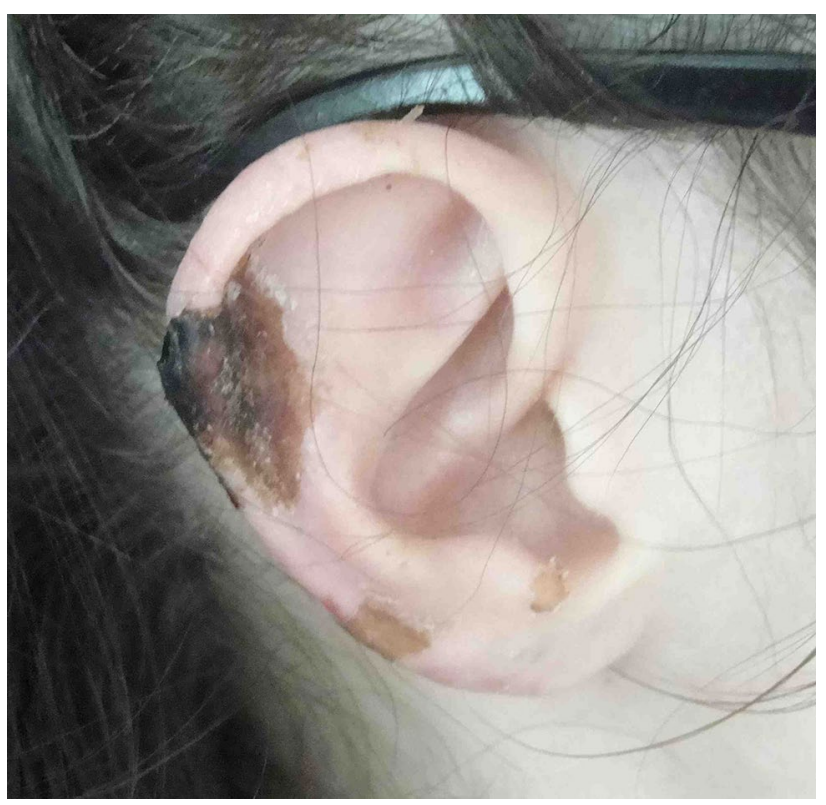

Fig. 1 Stage I: violaceous maculopapular plaques, net-like/reticular pattern 


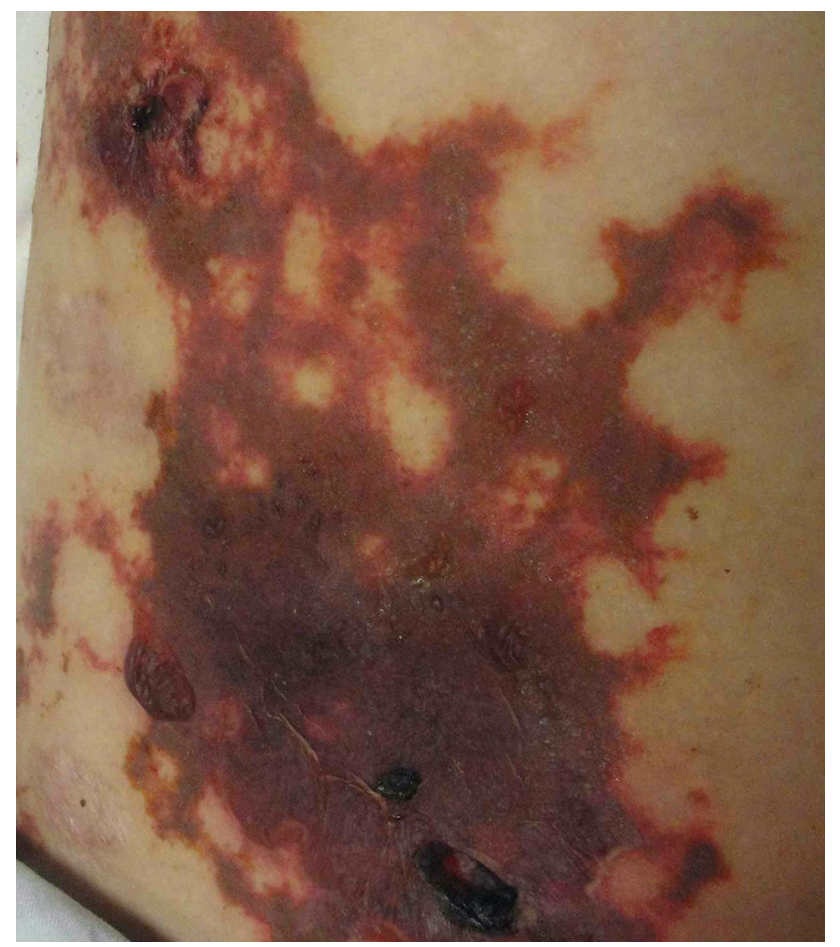

Fig. 2 Stage II: progression to bullae, ulceration, skin sloughing, and necrosis

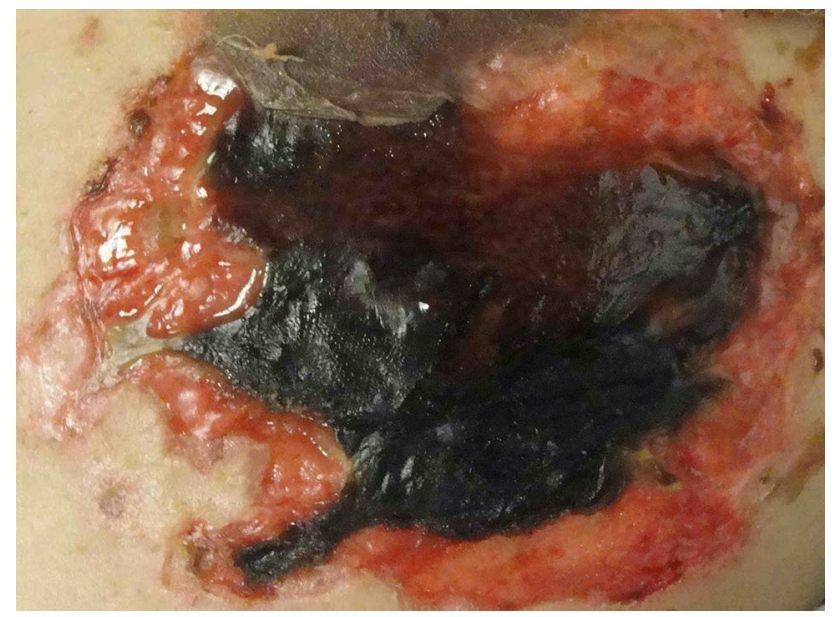

Fig. 3 Stage III: revelation of underlying fascia, tendon, adipose tissue, and muscle always progress to more severe stages. It is common for such lesions to spontaneously resolve upon cessation of cocaine, and therefore levamisole. Upon repeat exposure, lesions tend to progress to more severe stages at increasing rates [21, 22].

In addition to cutaneous symptoms, arthralgias and constitutional symptoms are common. In one case series by McGrath et al. [23], 72\% of patients reported at least one constitutional symptom including fever, night sweats, weight loss, or malaise, and $83 \%$ of patients reported arthralgias. In another case series by Munoz et al. [24], 23\% of patients reported fever and $40 \%$ reported arthralgias.

In the largest systematic review to date, Darteval et al. [7] reviewed 192 cases of levamisole-induced vasculitis. While $94.8 \%$ of symptomatic patients had cutaneous manifestations, 12.5 and $10.4 \%$ presented with acute kidney injury or pulmonary disease, respectively. Among patients with acute kidney injury, renal biopsy most commonly demonstrated crescentic glomerulonephritis. Of these patients, $41.7 \%$ recovered, and $29.2 \%$ went on to develop chronic renal insufficiency. Among patients with pulmonary involvement, alveolar hemorrhage and pneumonia were the most common presentations. One patient died of necrotizing pneumonia. In other case reports describing pulmonary involvement, patients presented with shortness of breath and imaging abnormalities. Chest X-ray demonstrated pulmonary infiltrates and chest computed tomography demonstrated ground-glass opacities [7, 23-26]. An overview of the systematic manifestations of levamisole-induced vasculitis is described in Table 2.

\section{Pathogenesis}

Pathogenesis is thought to be due to a combination of direct toxicity to neutrophils and endothelial cells as well as the loss of tolerance to self-antigens leading to autoimmunity [32]. The mechanism by which these autoantibodies develop is not fully understood, but recent reports have implicated neutrophil extracellular traps (NETs) [27-29].

NETs are collections of mitochondrial DNA bound to neutrophil granule proteins such as neutrophil elastase and myeloperoxidase. Under normal conditions, NETs are released by neutrophils in response to bacterial infection or other physiologic stressors. Their high mitochondrial DNA content stimulates cytokine production for a regulated host-defense response [36].

Levamisole can directly induce the release of NETs from neutrophils via interaction with muscarinic receptors [34]. This excessive release of NETs causes neutrophil granule
Table 1 Stages of levamisoleinduced retiform purpura
Retiform purpura: violaceous maculopapular plaques, net-like/reticular pattern Progression to bullae, ulceration, skin sloughing or necrosis

Full-thickness necrosis, revelation of underlying soft tissue (e.g., fascia, tendon, adipose tissue, muscle) and/or bone 
Table 2 Systemic manifestations of levamisoleinduced vasculitis

\begin{tabular}{ll} 
Renal & $\begin{array}{c}\text { Necrotizing and crescentic pauci-immune glomerulonephritis, [1, 20, } \\
\text { 27] membranous nephropathy, [1, 27, 28] interstitial nephritis [27] }\end{array}$ \\
Pulmonary & Alveolar hemorrhage, [20] pulmonary hypertension, [1] serositis [24] \\
Neurological & Multifocal inflammatory leukoencephalopathy, [29] seizures [24] \\
Musculoskeletal & Acute arthritis, chronic deforming seronegative arthritis [30] \\
Gastrointestinal & Acute pancreatitis, [31] hepatosplenomegaly [24] \\
\hline
\end{tabular}

contents to act as self-antigens. Processing by antigen-presenting cells leads to the formation of anti-neutrophil autoantibodies directed against granule contents (ANCAs). ANCAs then stimulate neutrophils, further promoting the release of NETs [33]. NETs released into vasculature cause direct toxicity to endothelial cells, impair vasorelaxation, and cause intravascular thrombosis [33, 34]. Binding of ANCAs to NETs may trigger complement or Fc receptor-mediated inflammation, further contributing to their immunogenicity [35].

Understanding the molecular mechanism for the formation of NETs has implications for targeted therapies in the future. Recently, Sofoluwe et al. [37] demonstrated that ATP and the ATP channel pannexin1 (Panx1) contribute to NET formation. Panx $1 \mathrm{KO}$ mice and WT mice with Panx 1 channel inhibition showed a reduction in NET production, making these potentially useful therapeutic targets for the future.

\section{Diagnosis}

The presence of retiform purpura in the setting of cocaine use should raise high suspicion for levamisole exposure. An accurate drug history may be difficult to obtain, but the diagnosis may still be made in the context of negative cocaine and/or levamisole testing. Sensitivity for detecting cocaine via urine toxicology decreases $48 \mathrm{~h}$ after use [38].Sensitivity for detecting serum or urine levamisole via liquid and gas chromatography-mass spectrometry diminishes $48-72 \mathrm{~h}$ after exposure [38].

The differential diagnosis for retiform purpura is broad and includes vasculitic, coagulopathic, viral, bacterial, autoimmune, and embolic etiologies as summarized in
Table 3. Infectious, coagulopathic, and embolic etiologies should be excluded first as they represent potential emergent situations [22].

Once the differential is narrowed and vasculitis is suspected, serologic studies for autoantibodies, especially ANCAs, should be performed [22]. In patients presenting with skin lesions, 95-100\% have positive ANCA serologies and of those over $90 \%$ demonstrate a p-ANCA predominant pattern [20, 25]. P-ANCA titers are exceptionally elevated compared to those seen in other vasculitides. Antibodies are generally directed against multiple, atypical antigens such as human neutrophil elastase (HNE), lactoferrin, and cathepsin G [20]. When present, p-ANCA antibodies directed against myeloperoxidase, its typical target, are found concomitantly with antibodies targeting multiple components of neutrophil granules. These finding are helpful in differentiating levamisole-induced vasculitis from other ANCAassociated vasculitides [1, 20]. Notably, the presence of p-ANCA directed against HNE is highly specific for levamisole-induced vasculitis [17]. When present, c-ANCA directed against proteinase antigen 3 appears at lower titers than in other vasculitides. The majority of patients are also positive for antiphospholipid antibodies including lupus anticoagulant and anticardiolipin [25, 44]. Positivity for antinuclear and anti-double-stranded DNA antibodies has been reported at varying frequencies [32].

Hematologic abnormalities occur in approximately $60 \%$ of patients with cutaneous symptoms [20]. Neutropenia and agranulocytosis are characteristic features, typically occurring 1-2 weeks after initial exposure [1]. Plasmacytoid lymphocytes in peripheral blood, bone marrow plasmacytosis, and megakaryocyte hyperplasia have been described

Table 3 Differential diagnosis of retiform purpura [39, 40]

Vasculitides: granulomatosis with polyangiitis (Wegener's granulomatosis), polyarteritis nodosa, eosinophilic granulomatosis with polyangiitis (Churg Strauss syndrome), [41] microscopic polyangiitis, cryoglobulinemia, erythema multiforme, levamisole-induced vasculitis

Coagulopathies: warfarin necrosis, heparin necrosis, purpura fulminans, immune thrombocytopenic purpura, disseminated intravascular coagulation

Viral: HIV, hepatitis B \& C, HHV-8 Kaposi's sarcoma, [42] SARS-CoV-2 [13]

Bacterial: bacterial sepsis due to staphylococci, streptococci, vibrio, M. morganii, [43] ecthyma grangrenosum, acute meningococcemia, Rickettsial disease

Vascular: calciphylaxis

Autoimmune: toxic epidermal necrolysis, systemic lupus erythematosus, antiphospholipid syndrome

Embolic: septic, fat, air, cholesterol, marantic 
in patients with agranulocytosis [1]. Other common findings include elevated erythrocyte sedimentation rate, elevated serum C-reactive protein, hypocomplementemia, elevated APTT and PT, and low protein C and S levels [17, 20, 32].

Histopathology of skin biopsy specimens typically reveals leukocytoclastic vasculitis and/or thrombotic vasculopathy in small-to-mid-sized vessels. Leukocytoclastic vasculitis may or may not be associated with fibrinoid necrosis [7, 17, 23]. Common findings include epidermal necrosis, dermal inflammation and hemorrhage, and thrombosis within dermal vessels [21]. Subepidermal edema, erythrocyte extravasation, and visualization of neutrophils in blood vessel walls have also been described [22]. Direct immunofluorescence has not been routinely performed, but in a series of 21 biopsies undergoing IF assay, 16 (76.2\%) were positive for immunoglobulins and/or complement deposition [7].

\section{Treatment and prognosis}

In the majority of cases, cessation of cocaine and therefore levamisole results in rapid improvement of cutaneous lesions within 2-3 weeks. Serological markers typically return to normal between 2 and 14 months, while neutrophil levels normalize after 5-10 days [17].

Continued use of cocaine is associated with progression of disease and poorer outcomes. Patients should be counseled on their cocaine use and advised that continued exposure or re-exposure could lead to more severe symptoms with faster onset in the future. Interventions to address patient substance-use disorders have led to improved short- and long-term outcomes for patients [45].

For symptomatic relief of pain, non-steroidal anti-inflammatory drugs including aspirin have been effective, especially in those with severe arthralgias [46]. Colchicine and oral antihistamines may also provide symptomatic relief [17].

For patients whose symptoms do not improve with cocaine cessation alone, and for those with systemic manifestations, oral steroids and other immunosuppressants may be effective [46]. Though systemic steroids have been routinely employed in all patients, their effect is unclear outside of systemic involvement or refractory cases [47]. Steroids should be used with caution due to the potential for adverse effects and immunosuppression, especially in patients with severe neutropenia. In cases of severe systemic involvement, dapsone, pentoxifylline, intravenous immunoglobulin, plasmapheresis, mycophenolate mofetil, cyclosporine, and other immunosuppressants have been utilized, although their efficacy is unclear [17]. Rituximab, though frequently used in the management of other ANCA-associated vasculitis, has been rarely used in the treatment of levamisole-induced vasculitis. This may be due to the fact that it is recognized for causing a drug-induced cutaneous vasculitis itself. [48].

Patients with agranulocytosis or symptomatic neutropenia should be hospitalized and started on intravenous, broadspectrum antibiotics. Granulocyte colony-stimulating factor may be considered for these patients if there is evidence of infection or if patients suffer from comorbid immunosuppression, such as in HIV-positive patients [19].

Patients with stage III skin lesions may require hospitalization, as wound debridement, skin grafts, and amputation may be necessary. The decision of when to operate is based on clinical judgement. Mcevenue et al. [49] found that early excision in the setting of ongoing purpura was associated with disease progression involving greater total body surface areas, possibly due to the pro-inflammatory effects of surgery [49]. They recommend initial excision to be performed after lesions are fully demarcated. Initial debridement should include fascial excision to reduce the need for additional operations. Earlier excision may be appropriate in the setting of wound sepsis. Following excision, staged grafting has led to improved patient outcomes [49].

\section{Epidemiology and clinical diagnosis of COVID-19-induced retiform purpura}

\section{Epidemiology}

While the true incidence of cutaneous symptoms in COVID19 patients is evolving, a study by Recalcati [50] found that number to be over $20 \%$. A variety of dermatologic morphologies have been described, of which retiform purpura is among the most common [13]. In the largest case series of COVID-19-induced retiform purpura to date, Freeman et al. [13] found that the average presenting age was 66 and lesions were more common in males.

\section{Clinical presentation}

COVID-19 patients presenting with retiform purpura are typically critically ill. In the case series by Freeman et al. [13], $100 \%$ of patients were hospitalized, $82 \%$ presented with ARDS, and $91 \%$ required a ventilator and/or ECMO. This is consistent with other case series [10, 51-53]. Lesions typically present after the initial onset of COVID-19 symptoms and most commonly involve the hands and feet [8, $13,45,52,53]$. Lesions tend to be painless, but have been described as progressing to painful ischemia and necrosis $[13,51]$. As of yet, no staging system for COVID-19-induced retiform purpura has been described. We suggest the staging system we propose for levamisole-induced retiform purpura may be valid. 
In addition to the pulmonary manifestations, patients with COVID-19-induced retiform purpura are also commonly present with fever, malaise, and arthralgias [13, 51]. The most common complications seen in these patients are thrombotic events, including stroke and pulmonary embolism, as well as acute kidney injury [13, 51-53].

Pathogenesis of COVID-19-induced retiform purpura is thought to be due to a pauci-inflammatory thrombogenic vasculopathy seen exclusively in critically ill patients. Magro et al. [52] attributed the thrombogenic vasculopathy to a blunted type 1 interferon response, which is needed for viral elimination. Depressed interferon signaling leads to extensive viral replication and viral protein release. Viral proteins are then thought to localize to vascular endothelial cells, leading to activation of the mannan-binding lectin and alternative complement pathways. Complement activation and deposition leads to extensive vascular injury and thrombosis [52].

\section{Diagnosis}

The differential diagnosis for COVID-19-induced retiform purpura includes other etiologies of retiform purpura, as summarized in Table 3.

For symptomatic patients in whom suspicion for COVID19 exists, the Infectious Diseases Society of America (IDSA) recommends direct SARS-CoV-2 nucleic acid amplification testing of nasal specimens. Repeat testing following negative results is warranted when clinical suspicion remains high [54].

Histopathology of skin biopsy specimens consistently shows pauci-inflammatory thrombogenic vasculopathy with or without complement deposition in capillaries, venules, and arteries [11, 13, 51-53]. Patients often have an elevated D dimer level, suggesting an underlying prothrombotic state [51-53]. Normal-to-elevated fibrinogen, normal haptoglobin, and an absence of thrombocytopenia or schistocytes have been used to rule out DIC or thrombotic microangiopathy which has also been described in critically ill COVID-19 patients $[10,51,53,55]$.

\section{Treatment and prognosis of COVID-19-induced retiform purpura}

Patients with COVID-19-induced retiform purpura are typically ill with a poor prognosis. Mortality ranges between 10 and $50 \%[13,52,53,56]$. While effective treatment for COVID-19 continues to evolve, the use of anticoagulants in those presenting with retiform purpura has shown some success [11]. In several cases, despite starting a prophylactic dose of anticoagulation at presentation, patients later required therapeutic doses due to ongoing thrombosis [11]. In at least one case, anticoagulation was continued weeks after hospital discharge, with progressive resolution of cutaneous lesions [51].

Other treatment modalities commonly employed in severe COVID-19 such as antimalarials, antivirals, antibiotics, IL-6 inhibitors, and corticosteroids have been used at varying frequencies $[13,51,56]$. As of yet, no data comparing their relative effectiveness in treating COVID-19-induced retiform purpura have been published to our knowledge.

Severe COVID-19 has been linked to two scenarios: (1) a delayed induction of beneficial cytokines, especially type 1 interferons and (2) an overabundance of detrimental cytokines such as IL-6, otherwise known as the "cytokine storm" [57]. As previously discussed, COVID-19-induced retiform purpura appears to result from an inadequate type 1 interferon response. Therefore, immunosuppressants such as IL-6 inhibitors and corticosteroids, which are predominantly used in the context of cytokine storm, should be used with caution, if at all [57, 58]. Furthermore, IL-6 inhibitor antibodies have been linked to delayed thrombus resolution, further suggesting a limited role in the treatment of COVID-19-induced retiform purpura [59]. The administration of type I interferon as an effective therapeutic agent in severe COVID19 is currently being explored in multiple clinical trials [57]. Currently, the NIH COVID-19 Treatment Guidelines Panel recommends against the use of interferons in severe COVID-19, except within ongoing clinical trials [60]. Additional well-designed studies evaluating the use of interferons in those with COVID-19-induced retiform purpura are anticipated.

A comparison of the clinical features of COVID-19-induced retiform purpura and levamisole-induced vasculitis is summarized in Table 4.

\section{Conclusions}

Levamisole-induced and COVID-19-induced retiform purpura are two syndromes with overlapping clinical features. Accurate distinction between the two has significant clinical implications. Whereas one syndrome is primarily an autoimmune disease treated with immunosuppression, the other results from an inadequate immune response and is treated with anticoagulation. At the present time, the possibility that patients may present with one or both syndromes creates as diagnostic challenge. As the pandemic progresses, cases of COVID-19 continue to abound. At the same time, new psychosocial stressors produced by the pandemic are expected to increase rates of substance abuse. For a patient with retiform purpura 
Table 4 Clinical comparison of levamisole-induced and COVID-19-induced retiform purpura

\begin{tabular}{lll}
\hline & Levamisole-induced retiform purpura & COVID-19-induced retiform purpura \\
\hline Epidemiology & F $>$ M & M $>$ F \\
& Average age 42.7 & Average age 66 \\
Presentation & Cutaneous: retiform purpura (typically painful) & Cutaneous: retiform purpura (typically painless) \\
& Constitutional: fever, night sweats, weight loss, malaise & Constitutional: fever, malaise, \\
& Pulmonary: alveolar hemorrhage, pneumonia & Pulmonary: cough, shortness of breath, ARDS \\
& Renal: acute kidney injury & Renal: acute kidney injury \\
& MSK: arthralgias & MSK: arthralgias \\
& & Hematologic: thrombotic events (stroke, PE) \\
Histopathology & Leukocytoclastic vasculitis \pm fibrinoid necrosis & Pauci-inflammatory thrombogenic vasculopa- \\
& Thrombotic vasculopathy & thy \pm complement deposition \\
Laboratory findings & \pm cocaine/levamisole testing & +SARS-CoV-2 testing \\
& ANCA + & Elevated d dimer, elevated acute phase reactants \\
Treatment & Neutropenia/agranulocytosis & Anticoagulation \\
& Cocaine cessation & \\
NSAIDs & Steroids & \\
Prognosis & Surgical excision, grafting, amputation & \\
\hline
\end{tabular}

and respiratory symptoms, testing for SARS-CoV-2 and screening for cocaine and/or levamisole may be helpful, but are not absolute. A patient with levamisoleinduced vasculitis may incidentally test positive for COVID-19, and, conversely, a patient with COVID-19-induced retiform purpura may also use cocaine. An accurate diagnosis may rely on the clinical clues and distinguishing features outlined delineated herein.

Funding There is no funding sources.

\section{Declarations}

Conflict of interest Dr. Keim: nothing to disclose; Dr. Schwartz: nothing to disclose; Dr. Kapila: nothing to disclose.

Ethical approval This article does not contain any studies with human participants performed by any of the authors.

\section{References}

1. Cascio MJ, Jen KY (2018) Cocaine/levamisole-associated autoimmune syndrome: a disease of neutrophil-mediated autoimmunity. Curr Opin Hematol 25:29-36

2. Hofmaier T, Luf A, Seddik A et al (2014) Aminorex, a metabolite of the cocaine adulterant levamisole, exerts amphetamine like actions at monoamine transporters. Neurochem Int 73:32-41

3. Tallarida CS, Tallarida RJ, Rawls SM (2015) Levamisole enhances the rewarding and locomotor-activating effects of cocaine in rats. Drug Alcohol Depend 149:145-150
4. The Drug Enforcement Administration. National Drug Threat Assessment 2017, October 2017 edn. U.S. Drug Enforcement Administration, Springfield, VA

5. Brackney MD (2009) Morb Mortal Wkly Rep 58(49):1381-1385

6. Chung C, Tumeh PC, Birnbaum R et al (2011) Characteristic purpura of the ears, vasculitis, and neutropenia-a potential public health epidemic associated with levamisole-adulterated cocaine. J Am Acad Dermatol 65(4):722-725.e2

7. Dartevel A, Chaigne B, Moachon L et al (2019) Levamisoleinduced vasculopathy: a systematic review. Semin Arthritis Rheum 48(5):921-926

8. WHO Director-General's opening remarks at the media briefing on COVID-19. World Health Organization (WHO) https://www.who. int/director-general/speeches/detail/who-director-general-s-openi ng-remarks-at-the-media-briefing-on-covid-19---3-august-2020

9. Wiersinga WJ, Rhodes A, Cheng AC, Peacock SJ, Prescott HC (2020) Pathophysiology, transmission, diagnosis, and treatment of coronavirus disease 2019 (COVID-19): a review. JAMA 324(8):782-793. https://doi.org/10.1001/jama.2020.12839

10. Almutairi N, Schwartz RA (2020) COVID-19 with dermatologic manifestations and implications: an unfolding conundrum. Dermatol Ther 33(5):e13544. https://doi.org/10.1111/dth.13544

11. Young S, Fernandez AP (2020) Skin manifestations of COVID19. Clev Clin J Med. https://doi.org/10.3949/ccjm.87a.ccc031

12. Suchonwanit P, Leerunyakul K, Kositkuljorn C (2020) Cutaneous manifestations in COVID-19: lessons learned from current evidence. J Am Acad Dermatol 83(1):e57-e60. https://doi.org/ 10.1016/j.jaad.2020.04.094

13. Freeman EE, McMahon DE, Lipoff JB et al (2020) The spectrum of COVID-19-associated dermatologic manifestations: an international registry of 716 patients from 31 countries. J Am Acad Dermatol 83(4):1118-1129. https://doi.org/10.1016/j.jaad.2020. 06.1016

14. World Health Organization. (2020) Novel Coronavirus (2019$\mathrm{nCoV}$ ): situation report, 209. World Health Organization. https:// www.who.int/docs/default-source/coronaviruse/situation-reports/ 20200816-covid-19-sitrep-209.pdf?sfvrsn=5dde1ca2_2 
15. Dubey MJ, Ghosh R, Chatterjee S, Biswas P, Chatterjee S, Dubey S (2020) COVID-19 and addiction. Diabetes Metab Syndr 14(5):817-823. https://doi.org/10.1016/j.dsx.2020.06.008

16. Wei Y, Shah R (2020) Substance use disorder in the COVID-19 pandemic: a systematic review of vulnerabilities and complications. Pharmaceuticals (Basel) 13(7):155. https://doi.org/10.3390/ ph13070155

17. Imbernón-Moya A, Chico R, Aguilar-Martínez A (2016) Cutaneous and mucosal manifestations associated with cocaine use. Med Clin (Barc) 146(12):544-549

18. Wolford A, McDonald TS, Eng H, Hansel S, Chen Y, Bauman J et al (2012) Immune-mediated agranulocytosis caused by the cocaine adulterant levamisole: a case for reactive metabolite(s) involvement. Drug Metab and Dispos 40(6):1067-1075

19. Brunt T, Berg J, Pennings E, Venhuis B (2017) Adverse effects of levamisole in cocaine users: a review and risk assessment. Arch Toxicol 91(6):2303-2313

20. Nolan AL, Jen KY (2015) Pathologic manifestations of levamisole-adulterated cocaine exposure. Diagn Pathol 10(1):48-48

21. George TC, Freet DJ, Cross JM, Huzar TF (2019) Levamisoleinduced vasculitis. JAAPA 32(1):23-27

22. Jin Q, Kant S, Alhariri J, Geetha D (2018) Levamisole adulterated cocaine associated ANCA vasculitis: review of literature and update on pathogenesis. J Community Hosp Intern Med Perspect 8(6):339-344

23. Mcgrath MM, Isakova T, Rennke HG et al (2011) Contaminated cocaine and antineutrophil cytoplasmic antibody-associated disease. Clin J Am Soc Nephrol 6(12):2799-2805

24. Muñoz-Vahos H, Herrera-Uribe A, Arbeláez-Cortés A et al (2019) Clinical profile of levamisole-adulterated cocaine-induced vasculitis/ vasculopathy: a 30-case series. J Clin Rheumatol 25(3):e16-e26

25. Agdamag AC, Gevorgyan O, Lawrenz Co M, Hassan S (2018) Multiple cutaneous and mucosal lesions in a patient with cocainelevamisole-induced vasculopathy syndrome. Proc (Bayl Univ Med Cent) 32(1):93-95

26. Ullrich K, Koval R, Koval E, Bapoje S, Hirsh J (2011) Five consecutive cases of a cutaneous vasculopathy in users of levamisoleadulterated cocaine. J Clin Rheumatol 17(4):193-196

27. Lötscher F, Krusche M, Ruffer N, Kubacki T, Person F, Kötter I (2019) Cocaine-induced ANCA-associated renal disease: a casebased review. Rheumatol Int 39(11):2005-2014

28. Collister D, Sathianathan C, Ryz K et al (2017) ANCA associated vasculitis secondary to levamisole-adultered cocaine with associated membranous nephropathy: a case series. Am J Nephrol 45(3):209-216

29. Vosoughi R, Schmidt BJ, Vosoughi R (2015) Multifocal leukoencephalopathy in cocaine users: a report of two cases and review of the literature. BMC Neurol 15(1):208-208

30. Emil SN, Cisneros RD, Penmetsa SH et al (2020) Atypical chronic inflammatory ANCA-positive deforming arthritis after cocainelevamisole exposure. J Clin Rheumatol 26(1):24-32

31. Ogunbameru A, Jandali M, Issa A et al (2015) Acute pancreatitis as initial presentation of cocaine-induced vasculitis: a case report. J Pancreas 16(2):192-194

32. Graf J (2013) Rheumatic manifestations of cocaine use. Curr Opin Rheumatol 25(1):50-55

33. Lood C, Hughes GC (2017) Neutrophil extracellular traps as a potential source of autoantigen in cocaine-associated autoimmunity. Rheumatology (Oxford) 56(4):638-643

34. Carmona-Rivera C, Purmalek MM, Moore E et al (2017) A role for muscarinic receptors in neutrophil extracellular trap formation and levamisole-induced autoimmunity. JCI Insight 2(3):e89780-e89780

35. Söderberg D, Segelmark M (2018) Neutrophil extracellular traps in vasculitis, friend or foe? Curr Opin Rheumatol 30(1):16-23
36. Yousefi S, Simon D, Stojkov D, Karsonova A, Karaulov A, Simon H-U (2020) In vivo evidence for extracellular DNA trap formation. Cell Death Dis 11(4):300-315

37. Sofoluwe A, Bacchetta M, Badaoui M, Kwak BR, Chanson M (2019) ATP amplifies NADPH-dependent and -independent neutrophil extracellular trap formation. Sci Rep 9(1):16556

38. Shea J (2012) Bioanalytical methods for quantitation of levamisole, a widespread cocaine adulterant. Clin Chem Lab Med 51(1):205-212

39. Georgesen C, Fox LP, Harp J (2020) Retiform purpura: workup and therapeutic considerations in select conditions. J Am Acad Dermatol 82(4):799-816

40. Georgesen C, Fox LP, Harp J (2020) Retiform purpura: a diagnostic approach. J Am Acad Dermatol 82(4):783-796a

41. Schwartz RA, Churg J (1992) Churg-Strauss syndrome. Brit J Dermatol 127:199-204

42. Schroedl L, Kim E, Hoffman MD (2020) Iatrogenic Kaposi sarcoma presenting as retiform purpura during treatment of bullous pemphigoid. J Am Acad Dermatol 6(3):247-249

43. Calado R, Relvas M, Brites MM, Cardoso JC (2019) Acute retiform purpura caused by Morganella morganii. BMJ Case Rep 12(12):e233344

44. Graf J, Lynch K, Yeh CL et al (2011) Purpura, cutaneous necrosis, and antineutrophil cytoplasmic antibodies associated with levamisole-adulterated cocaine. Arthritis Rheum 63(12):3998-4001

45. Raheemullah A, Melhem M, Andruska N (2020) Cocaine cessation for levamisole-induced vasculitis: treating the underlying disease. J Clin Rheumatol 26(8):e276-e278

46. Mandrell J, Kranc CL (2016) Prednisone and vardenafil hydrochloride for refractory levamisole-induced vasculitis. Cutis 98(2):E15-E19

47. Pearson T, Bremmer M, Cohen J, Driscoll M (2012) Vasculopathy related to cocaine adulterated with levamisole: A review of the literature. Dermatol Online J. https://doi.org/10.5070/D36G18R9CV

48. Grau RG (2015) Drug-induced vasculitis: new insights and a changing lineup of suspects. Curr Rheumatol Rep 17(12):1-10

49. Mcevenue G, Brichacek M, Logsetty S, Shahrokhi S (2017) Surgical management of levamisole-adulterated cocaine induced soft tissue necrosis: case study and treatment algorithm. J Burn Care Res 38(3):e638-e646

50. Recalcati S (2020) Cutaneous manifestations in COVID-19: a first perspective. J Eur Acad Dermatol Venereol 34(5):e212-e213. https://doi.org/10.1111/jdv.16387

51. Bosch-Amate X, Giavedoni P, Podlipnik S, Andreu-Febrer C, Sanz-Beltran J, Garcia-Herrera A, Alós L, Mascaró JM (2020) Retiform purpura as a dermatological sign of coronavirus disease 2019 (COVID-19) coagulopathy. J Eur Acad Dermatol Venereol 34(10):e548-e549

52. Magro C, Mulvey JJ, Laurence J, Sanders S, Crowson AN, Grossman M, Harp J, Nuovo G (2021) The differing pathophysiologies that underlie COVID-19-associated perniosis and thrombotic retiform purpura: a case series. Br J Dermatol 184(1):141-150

53. Droesch C, Do MH, DeSancho M, Lee EJ, Magro C, Harp J (2020) Livedoid and purpuric skin eruptions associated with coagulopathy in severe COVID-19. JAMA Dermatol 156(9):1-3

54. Hanson KE, Caliendo AM, Arias CA et al (2020) Infectious diseases society of america guidelines on the diagnosis of COVID-19. Clin Infect Dis. https://doi.org/10.1093/cid/ciaa760

55. Singh P, Schwartz RA (2020) Disseminated intravascular coagulation: a devastating systemic disorder of special concern with COVID-19. Dermatol Ther 33(6):e14053. https://doi.org/10.1111/ dth. 14053

56. Galván Casas C, Català A, Carretero Hernández G et al (2020) Classification of the cutaneous manifestations of COVID-19: a rapid prospective nationwide consensus study in Spain with 375 cases. Br J Dermatol 183(1):71-77. https://doi.org/10.1111/bjd.19163 
57. Jamilloux Y, Henry T, Belot A et al (2020) Should we stimulate or suppress immune responses in COVID-19? Cytokine and anticytokine interventions. Autoimmun Rev 19(7):102567. https://doi. org/10.1016/j.autrev.2020.102567

58. Nosaka M, Ishida Y, Kimura A et al (2020) Crucial involvement of IL-6 in thrombus resolution in mice via macrophage recruitment and the induction of proteolytic enzymes. Front Immunol 10:3150

59. Jalkanen J, Hollmén M, Jalkanen S (2020) Interferon beta-1a for COVID-19: critical importance of the administration route. Crit Care 24(1):335
60. COVID-19 Treatment Guidelines Panel. Coronavirus Disease 2019 (COVID-19) Treatment Guidelines. National Institutes of Health. Available at https://www.covid19treatmentguidelines.nih.gov/. Accessed 12 Jun 2021

Publisher's Note Springer Nature remains neutral with regard to jurisdictional claims in published maps and institutional affiliations. 